\title{
Design on Japanese Listening Mobile Learning System based on iOS Platform
}

\author{
Yajie Zhang \\ College of Foreign Languages, Bohai University, Jinzhou, 121013, China \\ 908063810@qq.com
}

Keywords: iOS platform; Japanese listening; mobile learning; system design; development environmen; interface design

\begin{abstract}
Modern mobile communication technology and mobile learning theory combined with the mobile learning, provides a new model for Japanese listening learning. This system is based on the researches on the iOS platform. First of all, research by Cocoa touch, Media application, the Core service and the Core OS consisting of four layers such as iOS platform layer structure; Secondly, design by the resource management, autonomous learning, entertainment and hearing system management and so on four big modules of software function; Then, the iOS development environment to build, including building steps and engineering structure; Finally, the interface design, including design method and main control. In this paper, the research results, solved the Japanese listening mobile learning system development, the key problems can be realized according to the actual development situation appropriate modification, in order to improve the development efficiency and quality of the system.
\end{abstract}

\section{Introduction}

Rapid development of economy and society, the communication between the two countries is more extensive, involving economic cooperation, science and technology exchange and cooperation, cultural exchanges and cooperation, military exchanges and cooperation, etc. Communication between China and Japan need to master specialized personnel in Japanese. After the reform and opening up, a lot of Japanese majors in the colleges and universities to open, great achievements have been made in the Japanese teaching, to cultivate a large number of specialized personnel in Japanese, for China's modernization and made great contribution to the development of communication between China and Japan. University of Japanese teaching goal is to cultivate the students' comprehensive application ability, especially listening and speaking skills, to make them in the future study, work and social activities can communicate effectively in Japanese, at the same time to improve their ability of autonomous learning, improve the comprehensive cultural quality, to meet the need of social development in our country and international communication. Traditional universities in Japanese listening teaching using "speak the word, put the tape, to answer", in the form of such a teaching mode just gives students listening exercises, rarely focus on the cultivation of students' listening ability, have not been fully mobilize students' initiative and autonomy, less any skill training.

Mobile learning is the modern mobile communication technology and mobile learning theory combined with the emergence of a new form of learning. Learners can at any time and any place, use of wireless mobile communication technology, wireless mobile communications equipment and wireless communications networks, education information, education resources and education services, communicate with others, as a new form of learning. Mobile learning changed people's form, that is, by formal learning extends to the informal learning, to provide a new form of lifelong learning. To carry out mobile learning in Japanese listening teaching in university based on students as the center, self-control learning progress, personalized learning way to explore a new way. Japanese listening mobile learning system based on the iOS platform design, learners can be anywhere at any time through the iOS terminal access to the illustrated, the combination of dynamic and static resource, to arouse learners' interest in learning Japanese, enhance the ability of 
autonomic learning, to strengthen college students' listening and speaking skills in Japanese, Japanese comprehensive application ability, improve college Japanese listening teaching.

\section{iOS Platform Hierarchy}

IOS is a mobile operating system developed by apple. It was originally designed for the iPhone, subsequently applied to the iPod touch, iPod and Apple TV, and other products. IOS like apple Mac OS X, belongs to a class of Unix business operating system. Initial system called the iPhone OS, because the device, iPhone, iPod touch are using iPhone OS, later renamed the iOS (iOS registered trademarks of Cisco network equipment operating system in the United States, apple renamed Cisco authorized).All iOS programming interface, tools, and resources contained in the iOS SDK, through the integrated development environment to complete the application development. The iOS platform consists of four layers, and each layer contains a number of components, through component to complete the corresponding functions. Platform hierarchy is shown in Fig. 1.

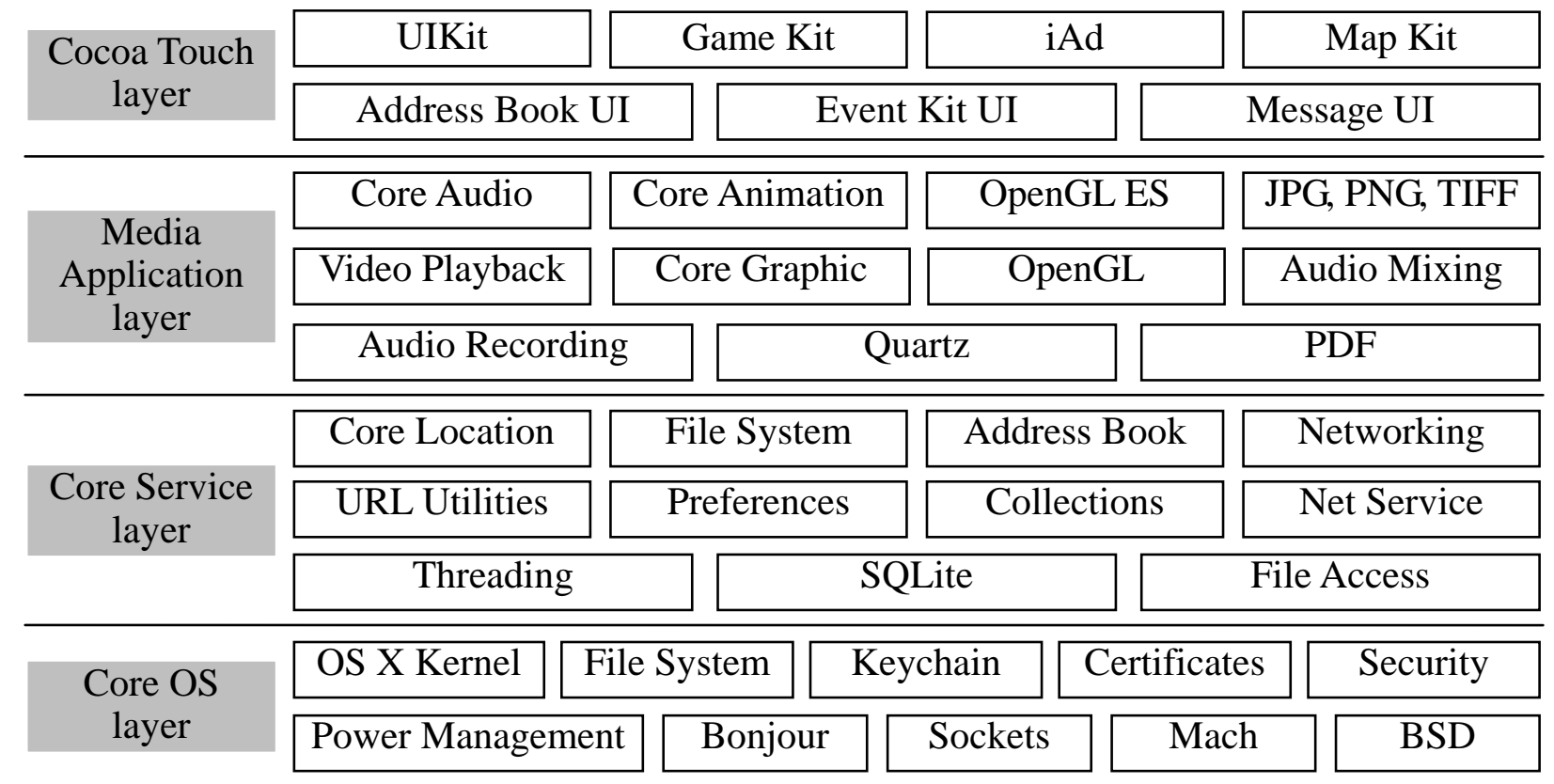

Fig .1. iOS platform Hierarchy

(1) Cocoa Touch layer. Cocoa Touch framework reuses a lot of Mac mature mode, to focus more on the Touch interface and optimization. UIKit provides on the iOS basic tools, graphics and event-driven programs based on as in Mac OS X on the Foundation of frame, including file processing, network and string manipulation, etc. Cocoa Touch with and the iPhone user interface is consistent with the special design. With UIKit, can use the iOS on unique graphical interface controls and buttons, as well as the full screen view function, you can also use the accelerometer and multi-touch gestures to control applications.

(2) The Media Application layer. Provide a complete all kinds of images, audio, and video and other multimedia services application and related technology development, application of the technology development will have a better visual and sound effects. IPhone OS high-level framework can be used to quickly create advanced graphics and animation. Among them, the Graphics Technologies, high quality image is an important part of all the iPhone app; Audio Technologies, including Audio playback, the quality of the recording and function of the vibration of the trigger device, etc.; Video Technologies, iPhone OS through the media player framework (MediaPlayer. Framework) supports full screen Video playback.

(3) The Core services layer (Core Service layer).Located in the Core OS layer above, provide the foundation for all application system service. AddressBook. Framework provides stored in mobile device in the phone book programming interface; CoreFoundation. The framework is based on $\mathrm{C}$ language interface set, providing basic data management and service function; CFNetwork. Framework provides object-oriented abstraction of the network protocols;. CoreLocation 
framework is used to obtain the present latitude and longitude of the mobile device; Security framework provides a management certificate, public/private key interfaces for and trust policy; SQLite is a small SQL database, you can create a local database files, and manage these tables and records in the file.

(4) The Core operating system layer (Core OS layer).The core OS layer contains the operating system kernel environment, drive and the basic interface. The kernel based on Mac operating system, is responsible for all aspects of the operating system. Virtual memory management systems, threads, file system, network and internal communication. Core OS layer driver also provides the interface between the hardware and system framework. IPhone OS provides many access interface low-level features of the operating system set, iPhone application through LibSystem libraries to access these functions, the interface set included: POSIX threads, BSD sockets, file system access, standard I/O, Bonjour and DNS services, Locale Information, memory allocation and mathematical calculation.

\section{System Function Design}

System function is the system has the efficiency and effect, system target through the system function to express and implement. Japanese listening mobile learning system for users, students, teachers and administrator user service, the system is mainly composed of resource management, autonomous learning, entertainment and hearing system management and so on four big modules, function structure is shown in Fig. 2.

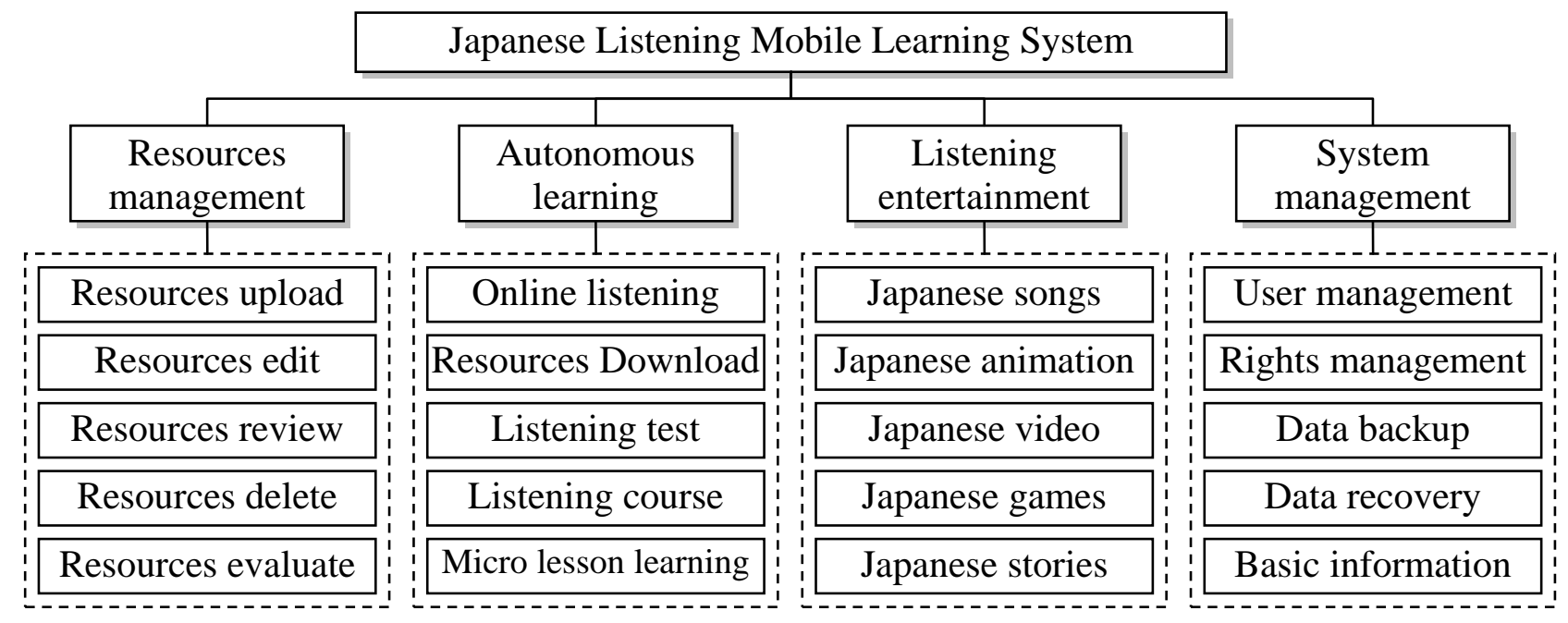

Fig. 2. Function Structure on Japanese Listening Mobile Learning System

In functional structure shown in Fig. 2, the user USES resource management mainly teachers, system management is mainly the system administrator users, in order to convenient and quick operation, is usually based on the Windows operating system on computer equipment is complete; Hearing entertainment and autonomous learning are mainly student users, is often used on iOS terminal. Resource management, mainly is the integrated management of resources, including the upload, edit, audit, delete, and evaluation, etc.; Autonomous learning, mainly for the students to complete the hearing, specific methods include online and offline hearing, as well as the test lesson, tutorials and learning, etc.; Hearing entertainment, mainly students in the process of entertainment and leisure reach the purpose of listening learning Japanese, including songs, animation, video, games and stories, etc.; System management, mainly is for system maintenance related work, including user management and permissions management, data backup and recovery as well as basic information maintenance, etc.

\section{iOS Development Environment to Build}

The development environment to build process as follows [6, 7]: download the installation package. 
Including download VMWare, Mac OS and the iPhone SDK installation package, download boot disk and registered Mac account;Install VMWare virtual machine. Including virtual machine VMWare Workstation installation, create a virtual machine and virtual machine Settings, etc.; In the virtual machine VMWare installed on Mac OS. With the mirroring Darwin and Iso guide, start the virtual machine, carried out in accordance with the prompt; The Shared directory. A way to install files carved into a CD, another way iphoneSDK directory Shared with VMWare the Mac; Install iphoneSDK. Found in the Mac iphoneSDK installation file, double-click the icon, according to the prompt operation; Start the Xcode began to develop. Open the Finder, double-click the Xcode icon, to create a new project, click the "Save" button to save. The structure of this system is shown in Table 1.

Table 1. iOS Engineering Structure

\begin{tabular}{|c|c|c|}
\hline No & Logical division & Instructions \\
\hline 1 & Application & $\begin{array}{l}\text { The AppDelegate of the UIApplicationDelegate protocol implementation } \\
\text { class constants and some system and the system configuration file }\end{array}$ \\
\hline 2 & Base & $\begin{array}{l}\text { ViewController parent and some public top-level custom parent class, } \\
\text { other modules classes generally inherit these classes }\end{array}$ \\
\hline 3 & Controller & $\begin{array}{l}\text { BaseViewController or BaseTableViewController which are inherited in } \\
\text { Group Base, realize the system control layer }\end{array}$ \\
\hline 4 & View & $\begin{array}{l}\text { System view, the view completely separated from the ViewController, can } \\
\text { keep view controllers downsizing }\end{array}$ \\
\hline 5 & Model & $\begin{array}{l}\text { Through the class to describe some roles in the system and the business, } \\
\text { at the same time contains corresponding to these roles and deal with the } \\
\text { business logic }\end{array}$ \\
\hline 6 & Handler & $\begin{array}{l}\text { The business logic layer, the whole system is responsible for processing } \\
\text { system of complex business logic, the upper caller is ViewController }\end{array}$ \\
\hline 7 & Storage & $\begin{array}{l}\text { Simple data storage, mainly some of the key/value pair and external files, } \\
\text { encapsulation of NSUserDefault and plist }\end{array}$ \\
\hline 8 & Network & $\begin{array}{l}\text { Network processing layer, encapsulating AFNetworking by block } \\
\text { implementation results callback, the upper invocation Handler layer }\end{array}$ \\
\hline 9 & Database & $\begin{array}{l}\text { Encapsulation FMDB sqlite and RTDatabaseHelper, provides the Model } \\
\text { layer object interface, encapsulating the stored procedure }\end{array}$ \\
\hline 10 & Utils & System tools, the main system commonly used tools \\
\hline 11 & Categories & The existing system and the expansion of the custom class \\
\hline 12 & Resource & Including images, animation, sound, and file resources \\
\hline
\end{tabular}

\section{Interface Design}

Interface design is mainly in the Xcode Interface Builder (IB).IB to help developers create UIView and added in the UIView interactive control, through the mouse to click mobile controls on the screen to set up the interface. The default controls include standard and custom mode control system controls. Standard system control including controller series, the series data view controls, input object and value object control series, and the window, view, and the control. Developers can use drag-and-drop to decide the position of each space, and use the inspector to set the properties of the controls and the correlation between control and program code. This system used in main control as shown in Table 2.

Control is the core of the iOS development work, the user interaction into the callback function to complete the process, so as to realize the software function. Control design includes two aspects, one is the attribute set, through the property list is complete; Second, the program design, program code to complete the incident response. User actions on the iPhone equipment mainly use touch to complete, so the UI interface of the control size and style need to conform to the touch, UI design 
proposal, according to apple should make software UI style matching equipment interface style, use as far as possible in line with the iOS interface style.

Table 2. System Used Main Control Description

\begin{tabular}{|c|c|c|}
\hline No & Control class & Effect \\
\hline 1 & UISwitch & Switch (instead of point selection) \\
\hline 2 & UIButton & Function button \\
\hline 3 & UISegmentedControl & The image display buttons peer distribution \\
\hline 4 & UISlider & Horizontal slide indicator \\
\hline 5 & UITextField & Text input \\
\hline 6 & UIPageControl & Control page \\
\hline 7 & UIToolBar & Toolbar (Show multiple icons and buttons) \\
\hline 8 & UTableView & Display data in the list \\
\hline 9 & UIImage & Display image \\
\hline 10 & UILabel & Label display text \\
\hline 11 & UINaviagtionBar & Navigation bar (to provide access to the shortcut) \\
\hline 12 & UIDatePicker & Date/time picker \\
\hline
\end{tabular}

\section{References}

[1] C. Jia, Y.S. Gao, Q. Gao, et al., "Reform and innovation of teaching methods of listening for Japanese majors," Journal of Shenyang Agricultural University(Social Sciences Edition), vol. 18, no. 2, pp. 225-228, 2016.

[2] X. J. Zhao, "Cultivation of intercultural communication awareness in Japanese listening comprehension," Journal of Changchun Education Institute, vol. 9, no. 4, pp. 78-80, 2007.

[3] waiwai's blog, "IOS development environment to build a utorial," http://blog.sina.com.cn/s/blog0102e246.html, 2017-1-20.

[4] lizhenmingdirk's Special column, "Build IOS development environment under Windows," http://blog.csdn.net/lizhenmingdirk/article/details/29850159, 2017-1-20.

[5] M. H. Li, "Design on instant communication message system of teachers and students for the iOS," Automation \& Instrumentation, vol. 35, no. 11, pp. 180-182, 2015.

[6] J. Shen, "Development of English learning video system based on iOS platform," Automation \& Instrumentation, vol. 36, no. 8, pp. 224-226, 2016. 